\title{
Clinical Characteristics of Respiratory Syncytial Virus Infections in Healthy Versus Previously Compromised Host
}

\begin{abstract}
Kathleen Meert, MD, Sabrina Heidemann, MD, Mary Lieh-Lai, MD, and Ashok P. Sarnaik, MD
Summary. In an effort to delineate the clinical characteristics of respiratory syncytial virus (RSV) infection in the compromised host, we compared children with bronchopulmonary dysplasia (BPD), congenital heart disease (CHD), premature birth, failure to thrive, and gastroesophageal reflux to previously healthy children. During a four-year period, 262 patients were admitted to the hospital with RSV infection diagnosed by a rapid RSV antigen detection test. Children with BPD or CHD had more hospital days and supplemental oxygen days than the previously healthy group $(P<0.05)$. Patients with BPD also had more ICU days, ventilator days, and NPO days, as well as a higher physiologic stability index and therapeutic intervention score than the previously healthy group $(P<0.05)$. Premature infants were more likely to present with apnea from RSV $(P<0.001)$. Patients with underlying illness tended to be older, although significant difference was demonstrated only for the BPD group $(7.0 \pm 5.3 \mathrm{vs.} 3.5 \pm 3.3, P<0.05)$. Patients with BPD and $\mathrm{CHD}$ had more nosocomial infections than the previously healthy group $(P<0.0001)$ and death occurred only in patients with underlying illness. We conclude that previously compromised patients are at risk for more severe and prolonged RSV disease. Earlier diagnosis and therapeutic intervention may be necessary in such patients to improve outcome. Pediatr Pulmonol. 1989; 7:167-170.
\end{abstract}

Key words: Hospital days; ventilator days; NPO-days; physiologic stability index; therapeutic intervention score; apnea; ribavirin therapy; nosocomial infections; mortality; risk factors.

\section{INTRODUCTION}

Respiratory syncytial virus (RSV) infection occurs in yearly outbreaks and is a major cause of upper and lower respiratory tract disease in young children. Between $5 \%$ and $40 \%$ of pneumonias and $60-90 \%$ of bronchiolitis in young children are caused by RSV, and virtually all children will have had at least one RSV infection by three years of age. ${ }^{1}$ The readily available rapid laboratory identification of the virus has helped understanding the epidemiology and mode of transmission of RSV disease. Significant morbidity and mortality have been attributed to RSV, particularly in children with previous cardiopulmonary compromise..$^{2-4}$ The purpose of this study was to evaluate the relationship between the previous state of health and the clinical characteristics of RSV disease in hospitalized children.

\section{MATERIALS AND METHODS}

The medical records of all patients hospitalized at Children's Hospital of Michigan with RSV infection for the four-year period between 1985 and 1988 were reviewed. The diagnosis was established by rapid RSV antigen detection test on nasal washings (Kallestad Path-
finder-Respiratory Syncytial Virus Direct Detection System). Nasal washings were obtained by the previously described technique of instilling sterile saline into the child's nares followed by suctioning with a rubber bulb syringe. ${ }^{5}$ Patients were allocated to one of the following six study groups based on their primary underlying diagnosis: 1) previously healthy group ( $\mathrm{HG}$ ) with no underlying medical problems; 2) history of bronchopulmonary dysplasia (BPD); 3) congenital heart disease (CHD); 4) prematurely born infants (PREM), with gestational age $\leq 37$ weeks, and no BPD or CHD; 5) failure to thrive (FTT) with body weight less than the fifth per-

From the Department of Pediatrics, Wayne State University School of Medicine, Children's Hospital of Michigan, Detroit, Michigan.

Received April 3, 1989; (revision) accepted for publication May 23, 1989.

This study was presented at the 54th Annual Symposium of the American College of Chest Physicians, Anaheim, California, October, 1988.

Address correspondence and reprint requests to Dr. A. Sarnaik, Critical Care Medicine, Children's Hospital of Michigan, 3901 Beaubien Boulevard, Detroit, MI 48201. 
centile for age; and 6) gastroesophageal reflux (GER) demonstrated on either nuclear scintiscan or esophageal $\mathrm{pH}$ determination.

Dependent variables measured included the age, number of hospital days, intensive care unit (ICU) days, ventilator days, supplemental oxygen days, NPO days, apnea at presentation, physiologic stability index (PSI), ${ }^{6}$ therapeutic intervention score (TISS), ${ }^{7}$ nosocomial infections, mortality, ribavirin use, and blood culture results. For patients on home oxygen therapy, supplemental oxygen days were defined as days when oxygen requirements were higher than the baseline home requirements. Apnea was defined as absence of respirations for longer than 15 seconds in hospital-observed cases or as absence of respirations associated with cyanosis in cases observed by a lay person prior to hospitalization. PSI is an established scoring system consisting of 34 variables from seven physiologic systems in which the degree of abnormality of each variable is assigned a standard score reflecting the clinical severity of the derangement. TISS consists of approximately 70 variables representing various therapeutic interventions each of which is assigned a standard score based on complexity and invasiveness of the therapy. Higher PSI scores indicate a greater degree of physiologic instability while higher TISS scores indicate more therapeutic intervention. The highest 24-hour scores for each patient were recorded. Nosocomial RSV infections were defined as those occurring in a previously uninfected child who had been hospitalized for more than seven days. ${ }^{8}$ Due to the retrospective nature of this study documentation of criteria for use of supplemental oxygen, ribavirin therapy, and the need for NPO could not be obtained and may have differed among the various clinical services caring for these patients. Mechanical ventilation was initiated for apnea, respiratory acidosis ( $\mathrm{pH}<7.25$ ), hypoxemia (capillary $\mathrm{PO}_{2}$ less than $50 \mathrm{~mm} \mathrm{Hg}$ at $\mathrm{FiO}_{2}$ of $60 \%$ ), or clinical impression of fatigue.

Statistical analysis was performed using chi-square test for nominal dependent variables and one-way analysis of variance followed by Tukey's Honestly Significant Difference test for discrete and continuous dependent variables.

\section{RESULTS}

During the four-year study period 262 patients were hospitalized with RSV infection. Of these, 19 had BPD, 13 had CHD, 56 were prematurely born without BPD or CHD, 8 had FTT, 4 had GER, and 161 had been previously healthy. Individual congenital heart lesions are summarized in Table 1 . One additional patient with asymptomatic human immunodeficiency virus infection acquired RSV-related upper respiratory tract illness in the hospital while awaiting foster care.

\section{TABLE 1-Types of Congenital Heart Disease}

\begin{tabular}{lc} 
Lesion & No. of patients \\
\hline Atrial septal defect & 3 \\
Ventricular septal defect & 2 \\
Atrial and ventricular septal defects & 3 \\
Atrioventricular canal & 2 \\
Hypoplastic right heart, pulmonary atresia & 2 \\
Mitrial regurgitation, left atrial aneurism & 1 \\
Total & 13 \\
\hline
\end{tabular}

The clinical characteristics of the hospital course in the various groups are described in Table 2. Because hospitalized patients are expected to be sicker than non-hospitalized patients, patients with nosocomial RSV infections $(n=12)$ were excluded from the analysis. Children with BPD were found to have significantly more hospital days, ICU days, ventilator days, supplemental oxygen days, and NPO days as well as a greater degree of physiologic instability and need for therapeutic intervention, than the HG $(P<0.05)$. The CHD group had more hospital days and supplemental oxygen days than $\mathrm{HG}(P$ $<0.05)$. CHD patients also tended to have higher although not statistically different PSI and TISS scores. The PREM group had more hospital days and higher PSI scores $(P<0.05)$. The FTT and GER groups did not differ from each other or from the HG in any of the above mentioned variables. Patients with pre-existing illness tended to be older than HG infants, although a significant difference was demonstrated only for those with BPD (7.0 \pm 5.3 months vs. $3.5 \pm 3.3$ months, $P<0.05$ ). Table 3 shows the proportion of patients in each group, excluding nosocomially acquired cases of RSV, requiring ICU admission, supplemental oxygen, mechanical ventilation, and NPO status.

Blood cultures were obtained in 129 patients during their RSV illness with only two yielding bacterial pathogens. One patient with CHD had enterococcal and one PREM had pneumococcal bacteremia.

The incidence of apnea as a presenting symptom, of the use of ribavirin, and of nosocomial infection are described in Table 4. Apnea occurred in 10\% (26/262) of all patients. Patients with PREM (11/56) and BPD (5/19) were more likely to have apnea as the initial manifestation of their RSV illness than the HG (7/161), $\mathrm{X}^{2}=$ $17.5, P<0.001$. Ribavirin therapy was employed in $13 \%(34 / 262)$ of all patients and most frequently $(54 \%$; $7 / 13$ ) in those with CHD. RSV was nosocomially acquired in 5\% (12/262) of all patients. More patients with BPD (4/19) and CHD (3/13) acquired nosocomial infection than $\mathrm{HG}$ patients $\left.(1 / 161), \mathrm{X}^{2}=35.4, P<0.0001\right)$. There were three deaths - two in the BPD and one in the CHD group. All deaths occurred because of severe, intractable hypoxia. Two of the three deaths resulted from nosocomial infection. 
TABLE 2-Clinical Characteristics of RSV Infection

\begin{tabular}{|c|c|c|c|c|c|c|c|c|}
\hline \multirow[b]{2}{*}{ Age } & \multirow[b]{2}{*}{ (mos) } & \multicolumn{2}{|c|}{ Hospital } & \multirow{2}{*}{$\begin{array}{c}\text { Ventilator } \\
\text { days }\end{array}$} & \multirow{2}{*}{$\begin{array}{c}\text { Supplemental } \\
\mathrm{O}_{2} \text { days }\end{array}$} & \multirow[b]{2}{*}{ NPO days } & \multirow[b]{2}{*}{ PSI } & \multirow[b]{2}{*}{ TISS } \\
\hline & & total days & ICU days & & & & & \\
\hline BPD & $7.0 \pm 5.3^{*}$ & $11.3 \pm 9.4^{*}$ & $3.9 \pm 7.8^{*}$ & $3.5 \pm 7.9^{*}$ & $7.4 \pm 9.9 *$ & $3.2 \pm 5.8^{*}$ & $10.3 \pm 11.1^{*}$ & $16.7 \pm 19.6^{*}$ \\
\hline CHD & $6.3 \pm 6.0$ & $9.1 \pm 3.6^{*}$ & $1.5 \pm 2.3$ & $0.2 \pm 0.6$ & $5.0 \pm 4.9^{*}$ & $1.3 \pm 1.9$ & $7.7 \pm 7.4$ & $11.9 \pm 8.9$ \\
\hline PREM & $4.8 \pm 5.8$ & $5.7 \pm 3.4^{*}$ & $0.9 \pm 2.1$ & $0.7 \pm 1.5$ & $2.3 \pm 3.4$ & $0.7 \pm 1.7$ & $6.5 \pm 5.9^{*}$ & $8.2 \pm 6.5$ \\
\hline FTT & $6.8 \pm 2.8$ & $4.1 \pm 1.7$ & $0.0 \pm 0.0$ & $0.0 \pm 0.0$ & $0.0 \pm 0.0$ & $0.0 \pm 0.0$ & $2.1 \pm 1.6$ & $4.1 \pm 2.3$ \\
\hline GER & $2.8 \pm 1.5$ & $4.3 \pm 2.5$ & $0.0 \pm 0.0$ & $0.0 \pm 0.0$ & $0.0 \pm 0.0$ & $0.0 \pm 0.0$ & $3.0 \pm 2.6$ & $3.7 \pm 0.6$ \\
\hline HG & $3.5 \pm 3.3$ & $4.1 \pm 2.5$ & $0.2 \pm 1.5$ & $0.1 \pm 1.0$ & $0.9 \pm 2.1$ & $0.3 \pm 1.1$ & $4.1 \pm 3.9$ & $5.7 \pm 4.5$ \\
\hline F & 3.9 & 14.6 & 6.3 & 7.0 & 12.7 & 7.1 & 6.0 & 8.6 \\
\hline$P$ & $<0.005$ & $<0.0001$ & $<0.0001$ & $<0.0001$ & $<0.0001$ & $<0.0001$ & $<0.0001$ & $<0.0001$ \\
\hline
\end{tabular}

${ }^{\mathrm{a}}$ All values in mean $\pm \mathrm{SD} . \mathrm{F}$ and $P$ values for one way analysis of variance across groups; all six groups compared to each other. *Significantly different from HG at 0.05 level by Tukey's Honestly Significant Difference Test.

TABLE 3-Number of Patients in Various Groups Requiring ICU Admission, Mechanical Ventilation, Supplemental Oxygen, and NPO Status ${ }^{a}$

\begin{tabular}{lcccc}
\hline & $\begin{array}{c}\text { ICU } \\
\text { admission }\end{array}$ & $\begin{array}{c}\text { Mechanical } \\
\text { ventilation }\end{array}$ & $\begin{array}{c}\text { Supplemental } \\
\text { oxygen }\end{array}$ & $\begin{array}{c}\text { NPO } \\
\text { status }\end{array}$ \\
\hline BPD & $5 / 15$ & $4 / 15$ & $8 / 15$ & $5 / 15$ \\
CHD & $4 / 10^{\text {b }}$ & $1 / 10$ & $6 / 10$ & $4 / 10^{\text {b }}$ \\
PREM & $12 / 55$ & $11 / 55^{\text {b }}$ & $24 / 55^{\text {b }}$ & $12 / 55$ \\
HG & $10 / 160$ & $5 / 160$ & $32 / 160$ & $18 / 160$ \\
$\mathrm{X}^{2}$ & 22.4 & 21.2 & 21.1 & 11.8 \\
$P$ & $<0.001$ & $<0.001$ & $<0.001$ & $<0.001$ \\
\hline
\end{tabular}

${ }^{\text {aFTT }}$ and GER groups not included in chi square analysis because of small sample size.

${ }^{b}$ Major component to chi square.

\section{DISCUSSION}

Children with CHD have been reported to develop severe illness with RSV infection. ${ }^{2}$ Our findings indicate that infants with BPD are also more susceptible to severe and prolonged illness from RSV than otherwise healthy children. The increased severity of illness is more likely to be due to additional cardiopulmonary compromise from RSV infection itself, rather than to secondary bacterial infections. The latter appears to be a rare complication of RSV infection even in the compromised host, as only two such patients were found to be bacteremic. Children with underlying cardiopulmonary illness, especially those with pulmonary hypertension, are particularly vulnerable to develop fatal RSV infection. ${ }^{1,2}$ The overall mortality in the present study $(1 \%)$ is lower than in other reported series, although all three deaths occurred in patients with pre-existing illness. Premature infants had a greater degree of physiologic instability, requiring more hospital days than HG. Also, the proportion of premature infants $(56 / 262,21 \%)$ was higher than expected from the observed prevalence of prematurity in the general population. Premature birth may therefore be an important risk factor for developing RSV infection of sufficient severity to require hospitalization.

Apnea is a known complication of RSV infection al-
TABLE 4-Number of Patients in Various Groups With Apnea, Ribavirin, and Nosocomial Infection ${ }^{2}$

\begin{tabular}{lccc}
\hline & Apnea & Ribavirin therapy & Nosocomial infection \\
\hline BPD & $5 / 19^{\mathrm{b}}$ & $5 / 19$ & $4 / 19^{\mathrm{b}}$ \\
CHD & $2 / 13$ & $7 / 13^{\mathrm{b}}$ & $3 / 13^{\mathrm{b}}$ \\
PREM & $11 / 56^{\mathrm{b}}$ & $10 / 56$ & $1 / 56$ \\
HG & $7 / 161$ & $12 / 161$ & $1 / 161$ \\
$\mathrm{X}^{2}$ & 17.5 & 26.2 & 35.4 \\
$P$ & $<0.001$ & $<0.001$ & $<0.0001$ \\
\hline
\end{tabular}

${ }^{\text {a}} \mathrm{FTT}$ and GER groups not included in chi square analysis because of small sample size.

bMajor component to chi square.

though the mechanism is unclear. It has been described as a form of central apnea, characterized by a lack of respiratory effort leading to cessation of ventilation. ${ }^{9}$ RSV-associated apnea has been reported to be most frequent during the first month of life and in premature infants, particularly if there is a history of apnea during the neonatal period. ${ }^{9,10}$ Our results indicate that prematurely born infants with or without BPD are at risk for apnea as a presenting feature of RSV infection.

The peak incidence of RSV infection necessitating hospitalization is two to five months of age. ${ }^{11}$ However, children with BPD may develop severe pulmonary compromise from RSV disease even at an older age. RSV should therefore be considered as a possible etiology of respiratory decompensation in BPD patients at any age. In this study population, CHD patients also tended to be at risk of developing RSV disease at an older age.

Nosocomial RSV infections are common. The risk of acquiring RSV while hospitalized during a community epidemic is high and proportional to the length of stay. ${ }^{8}$ We have demonstrated that nosocomial RSV infection is an important hazard for patients with BPD and CHD. The risks and benefits of elective admissions for BPD and CHD patients should therefore be carefully weighed during RSV epidemics. Protective isolation of such patients may decrease morbidity. 


\section{REFERENCES}

1. Hall CB. Respiratory syncytial virus. In: Mandell GL, Dougias RG, Bennett JE, eds. Principles and Practice of Infectious Diseases. New York, NY: John Wiley and Sons, 1985:877-888.

2. MacDonald NE, Hall CB, Suffin SC, Alexson C, Harris PJ, Manning JA. Respiratory syncytial viral infection in infants with congenital heart disease. N Engl J Med. 1982; 307:397-400.

3. Groothuis JR, Guitierrez KM, Lauer BA. Respiratory syncytial virus infection in children with bronchopulmonary dysplasia. Pediatrics. 1988; 82:199-203.

4. McMillan JA, Tristam DA, Weiner LB, Higgins AP, Sandstrom $\mathrm{C}$, Brandon R. Prediction of the duration of hospitalization in patients with respiratory syncytial virus infection: Use of clinical parameters. Pediatrics. 1988; 81:22-26.

5. Hall CB, Douglas RG. Clinically useful method for the isolation of respiratory syncytial virus. J Infect Dis. 1975; 131:1-5.

6. Yeh TS, Pollack MM, Ruttimann UE, Holbrook PR, Fields AI.
Validation of a physiologic stability index for use in critically ill infants and children. Pediatr Res. 1984; 18:445-451.

7. Keene AR, Cullen DJ. Therapeutic intervention scoring systems: Update 1983. Crit Care Med 1983; 11:1-3.

8. Hall CB, Douglas RG, Geiman JM, Messner MK. Nosocomial respiratory syncytial virus infections. N Engl J Med. 1975; 293: 1343-1346.

9. Church NR, Anas NG, Hall CB, Brooks JG. Respiratory syncytial virus-related apnea in infants. Am J Dis Child. 1984; 138: 247-250.

10. Bruhn FW, Mokrohisky ST, McIntosh K. Apnea associated with respiratory syncytial virus infection in young infants. J Pediatr. 1977; 90:382-386.

11. Parrott RH, Kim HW, Arrobio JO, Hodes DS, Murphy BR, Brandt CD, Camargo E, Chanock RM. Epidemiology of respiratory syncytial vinus infection in Washington, D.C. II. Infection and disease with respect to age, immunologic status, race and sex. Am J Epidemiol. 1973; 98:289-300. 